\title{
Ultraviolet emission of silicon quantum tips
}

\author{
W. H. Zheng, Jian-bai Xia, S. D. Lam, and K. W. Cheah ${ }^{\text {a) }}$ \\ Department of Physics, Hong Kong Baptist University, Kowloon Tong, Hong Kong, \\ People's Republic of China \\ M. R. Rakhshandehroo and S. W. Pang \\ Department of Electrical Engineering and Computer Science, The University of Michigan, Ann Arbor, \\ Michigan 48109-2122
}

(Received 1 July 1998; accepted for publication 12 November 1998)

\begin{abstract}
Silicon tips used as field emitters have dimensions that are within the quantum confinement regime. Therefore they can be considered as freestanding silicon tips. In this letter, a photoluminescence spectrum of a $100 \times 100$ array of silicon tips was taken at $10 \mathrm{~K}$. Narrow ultraviolet luminescence peaks were observed. Using the empirical pseudopotential homojunction model, it is demonstrated that these luminescence peaks come from energy levels arising from quantum confinement. By fitting the theoretical result to the experimental result, we conclude that the luminescence peaks come from Si quantum tips of about $20 \AA$ in width and that they are covered by silicon dioxide. (C) 1999 American Institute of Physics. [S0003-6951(99)03703-1]
\end{abstract}

Since Canham ${ }^{1}$ reported efficient $(>1 \%)$ room temperature photoluminescence (PL) in electrochemically etched $\mathrm{Si}$, there have been thousands of papers dedicated to porous $\mathrm{Si}$ light-emitting properties, the recombination mechanism, and possible device applications. In the past several years a whole new family of nanocrystalline Si-based materials ( $n c-\mathrm{Si}$ ) has been reproducibly prepared; these materials have shown light-emitting properties very similar to those in porous $\mathrm{Si}^{2}$ Most PL research is in the visible spectrum domain $(\sim 2 \mathrm{eV})$, and is aimed at applications in integrated optoelectronics and optical interconnects for advanced ultralarge-scale integration. Recently $\mathrm{Kim}^{3}$ observed visible and violet light emission at room temperature in silicon-rich oxide films deposited by electron cyclotron resonance plasma with a mixture of silane and oxygen. A sample shows four peaks at wavelengths of 363, 402, 475, and $557 \mathrm{~nm}$. The peaks show a blueshift as the temperature decreases, and the temperature coefficient is close to the value corresponding to the band gap of single crystalline Si. They concluded that the observed violet and blue emissions are due to direct transitions in confined $\mathrm{Si}$ nanocrystallites $(n c-\mathrm{Si})$.

In this letter we report on ultraviolet (UV) emission from a Si quantum tip array fabricated by electron beam lithography and dry etching. Details of the fabrication process are reported elsewhere. ${ }^{4,5}$ A scanning electron micrograph (SEM) image of the Si quantum tip array of $100 \times 100$ is shown in Fig. 1(a), and the field emitters have good size uniformity. The top of the tip dimension is $\leqslant 5 \mathrm{~nm}$, i.e., it is well inside quantum confinement dimensions [Fig. 1(b)]. Thus the electron band structure at the tip should exhibit the property of strong quantum confinement. The PL was taken with a $250 \mathrm{~mm}$ double monochromator that has a cooled photomultiplier tube (PMT) as a detector. The PMT has a flat response from 300 to $900 \mathrm{~nm}$. The sample was excited by the $325 \mathrm{~nm}$ line of a $\mathrm{He}-\mathrm{Cd}$ laser set at $15 \mathrm{~mW}$. The laser was

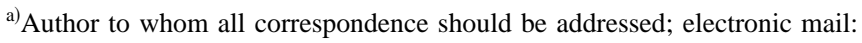
kwcheah@hkbu.edu.hk
}

focused to a spot size of $1 \mathrm{~mm}$ in diameter and the sample was cooled in a closed-cycle cryostat.

The UV PL spectrum of the array at $10 \mathrm{~K}$ is shown in Fig. 2. From Fig. 2 we see a broad emission in the UV spectrum range and on top of this broad spectrum there are several clear PL peaks located in the $3.1-3.3 \mathrm{eV}$ region. There is also a broad peak at $2.5 \mathrm{eV}$ and a shoulder at $3.0 \mathrm{eV}$. To the best of our knowledge it is the first report of ultraviolet emission of Si quantum tips. This emission is reproduc-

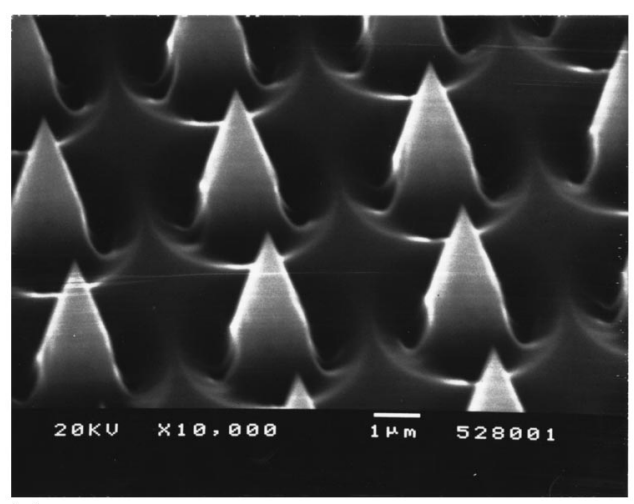

(a)

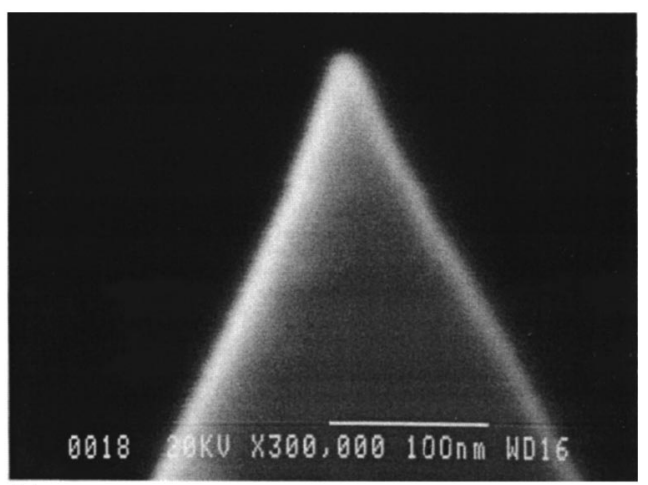

(b)

FIG. 1. (a) SEM of a $100 \times 100$ array of silicon tips. (b) High resolution SEM of the tip. The dimension at the top of the tip is estimated to be $\leqslant 5 \mathrm{~nm}$ (after Refs. 4 and 5). 


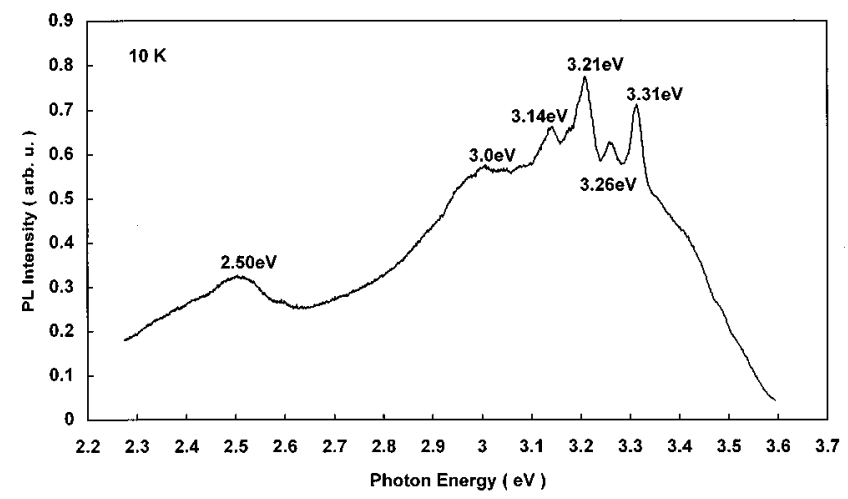

FIG. 2. PL spectrum of the silicon tip array at $10 \mathrm{~K}$. Four distinct peaks at energies of $3.31,3.26,3.21$, and $3.14 \mathrm{eV}$ were observed. A shoulder was observed at $3.0 \mathrm{eV}$ along with a broad peak at $2.50 \mathrm{eV}$.

ible by different arrays, and its wavelength is far shorter than visible light, which means there are potential applications in various fields.

We attribute the emission to the quantum confinement effect of the Si quantum tip. The quantum confinement effect in thin quantum wires has been discussed theoretically by the empirical pseudopotential homojunction model. ${ }^{6}$ Here we use this model to calculate the electronic states and optical transition matrix elements of Si quantum tips in a large energy range. The quantum tip is in the [001] direction, and assumed to have a square cross section. The normal vectors to the surfaces are along the [110] and [-110] directions. Hence the widths of the tip are $m$ and $n(\sqrt{2} a / 2)$, where $a$ is the lattice constant, and $m$ and $n$ are integers. Assuming that the surface of the Si tip is passivated by oxygen, the confinement potential is then taken as the band offset between $\mathrm{Si}$ and $\mathrm{SiO}_{2},{ }^{7} 3.2$ and $-4.6 \mathrm{eV}$ for the conduction and valence bands, respectively. Using this method we calculated the one-dimensional subbands $\left(-2 \pi / a<k_{z}<2 \pi / a\right)$ and the optical transition matrix elements,

$$
Q_{n n^{\prime}}^{i}=\frac{1}{m_{0}}\left|\left\langle n\left|p_{i}\right| n^{\prime}\right\rangle\right|^{2}, \quad i=x, y, z,
$$

and the lifetime,

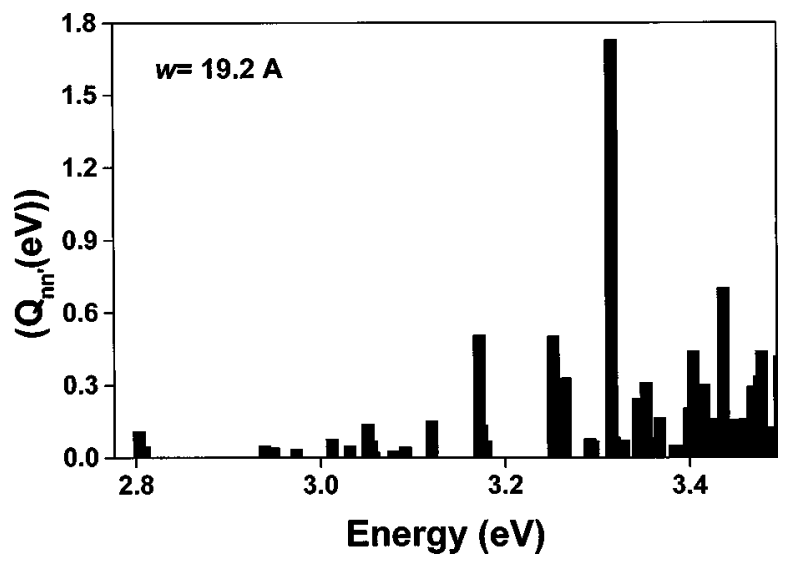

FIG. 3. Optical transition matrix elements as functions of transition energy for tips of width $(m, n)=(5,5)(19.2 \AA)$ and a surface passivated by $\mathrm{SiO}_{2}$. Transitions with transition matrix elements of less than $0.01 \mathrm{eV}$ are neglected. There are four strong transitions located at 3.17, 3.25, 3.27, and $3.32 \mathrm{eV}$.

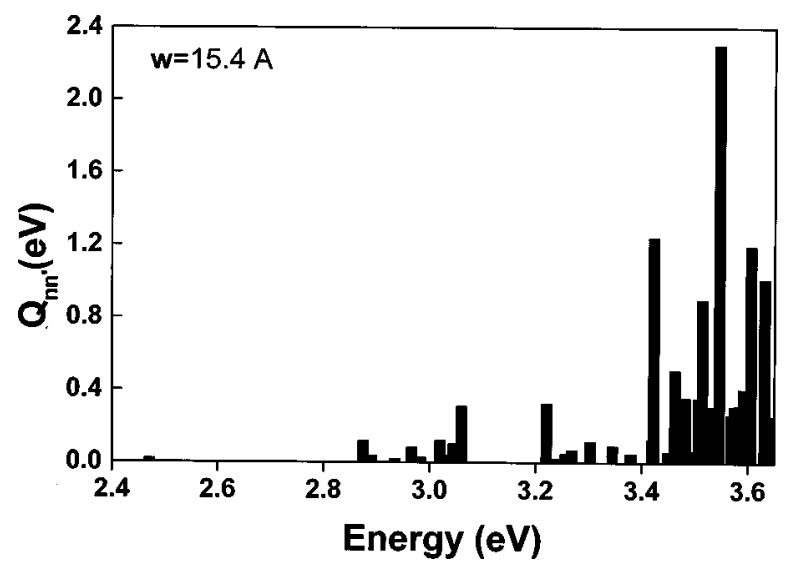

FIG. 4. Same as Fig. 3 except $(m, n)=(4,4)(15.4 \AA)$ and transitions with transition matrix elements of less than $0.01 \mathrm{eV}$ are also neglected. The four strong transitions are located at $3.42,3.46,3.51$, and $3.55 \mathrm{eV}$.

$$
\frac{1}{\tau}=\frac{4 \alpha \omega n}{3 m_{0} c^{2}} Q_{n n^{\prime}}
$$

for all possible transitions in the energy range of 2-4 eV, where $\alpha$ is the fine structure constant, $\omega$ is the photon angular frequency, and $n$ is the refractive index. The optical transition matrix elements as functions of transition energy for tips of width $(m, n)=(5,5)$ and $(4,4)(19.2$ and $15.4 \AA)$ are shown in Figs. 3 and 4, respectively. In Figs. 3 and 4 transitions with transition matrix elements of less than $0.01 \mathrm{eV}$ are neglected, therefore there are few transitions in the low energy range. When the energy is larger than $3.0 \mathrm{eV}$, there are increasingly more transitions with large transition matrix elements. For the case of width $(5,5)$ (Fig. 3) there are many transitions near $3.0 \mathrm{eV}$, corresponding to the PL shoulder at $3.0 \mathrm{eV}$ in Fig. 2. And there are also four strong transitions located at $3.17,3.25,3.27$, and $3.32 \mathrm{eV}$, corresponding to the PL peaks at $3.14,3.21,3.26$, and $3.31 \mathrm{eV}$, respectively, in Fig. 2. We believe the agreement is good. For the case of width $(4,4)$ (Fig. 4) there are also similar peak structures, but the transition energies shift to higher energy due to the thinner width. The four strong transitions are located at 3.42, $3.46,3.51$, and $3.55 \mathrm{eV}$. For comparison we made the same calculations for the case of a larger confinement potential: 8

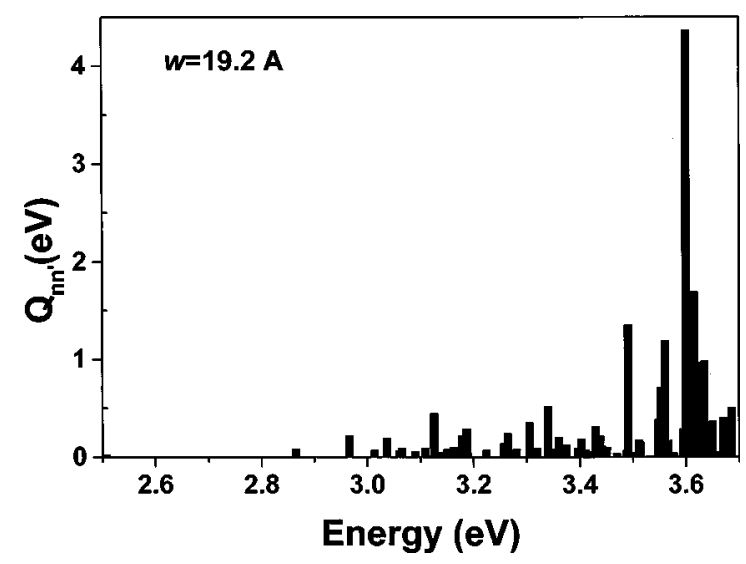

FIG. 5. Optical transition matrix elements as functions of transition energy for tips of width $m=5(19.2 \AA)$ and a surface passivated by $\mathrm{H}_{2}$. Transitions with transition matrix elements of less than $0.01 \mathrm{eV}$ are neglected. The four strong transitions are located at $3.31,3.34,3.43$, and $3.49 \mathrm{eV}$. 
TABLE I. Optical transition energies $\Delta E_{n n^{\prime}}$ (in units of $\mathrm{eV}$ ), transition matrix elements $Q_{n n^{\prime}}$ (in units of eV), and lifetimes $\tau$ (in units of $10^{-9} \mathrm{~s}$ ) for Si quantum tips with different confinement potentials $V_{0}$ (in units of $\mathrm{eV}$ ) and widths $w$ (in units of $\AA$ ).

\begin{tabular}{clllll}
\hline \hline$V_{0}=3.2,-4.6$ & $\Delta E_{n n^{\prime}}$ & 3.172 & 3.252 & 3.265 & 3.315 \\
$w=19.2$ & $Q_{n n^{\prime}}$ & 0.508 & 0.504 & 0.331 & 1.731 \\
& $\tau$ & 1.311 & 1.290 & 1.958 & 0.369 \\
$V_{0}=3.2,-4.6$ & $\Delta E_{n n^{\prime}}$ & 3.422 & 3.462 & 3.514 & 3.546 \\
$w=15.4$ & $Q_{n n^{\prime}}$ & 1.247 & 0.512 & 0.903 & 2.305 \\
& $\tau$ & 0.496 & 1.193 & 0.667 & 0.259 \\
$V_{0}=8.0,-6.0$ & $\Delta E_{n n^{\prime}}$ & 3.305 & 3.340 & 3.429 & 3.490 \\
$w=19.2$ & $Q_{n n^{\prime}}$ & 0.358 & 0.522 & 0.317 & 1.357 \\
& $\tau$ & 1.787 & 1.214 & 1.949 & 0.447 \\
\hline \hline
\end{tabular}

and $-6 \mathrm{eV}$ for the conduction and valence bands, respectively. This case simulates the surface atoms saturated by hydrogen atoms ${ }^{6}$ rather than by oxygen atoms. The optical transition matrix elements as functions of transition energy for tips of width $m=5$ are shown in Fig. 5, where the four strong transitions are located at 3.31, 3.34, 3.43, and 3.49 $\mathrm{eV}$. Due to the larger confinement potential, the transition energies increase by about $0.15 \mathrm{eV}$ relative to those of the earlier case. The transition matrix elements and lifetimes for all these transitions are given in Table I. From Table I we see that the lifetimes of the transitions are of the order of ns $\left(10^{-9} \mathrm{~s}\right)$.

The UV peaks on the top of the broad PL spectrum in Fig. 2 exhibit inhomogeneous broadening that is caused by size variation. To consider this effect, we calculated the optical transition matrix elements as functions of transition energy for tips of various widths. It was noticed that the size does not vary continuously from one layer to the next. ${ }^{8,9}$ The peak energy positions and optical transition matrix elements for tips of widths $(m, n)(\sqrt{2 a} / 2)$ are given in Table II. From Table II we see that for all tips except width $(4,4)$, the peak positions are clustered around four energy regions: 3.00, $3.18-3.20,3.25-3.26$, and $3.31 \mathrm{eV}$. When the width is equal to or less than $(4,4)$, the peak energy blueshifted to higher energy. This explains the small inhomogeneous broadening observed in Fig. 2. The results in Table I and Figs. 3-5 are obtained by summing all contributions from states of the one-dimensional subbands $\left(-2 \pi / a<k_{z}<2 \pi / a\right)$ and then averaging them, whereas the results in Table II are obtained from the contributions of $k_{z}$ states where $k_{z}=0$. In comparing the results for tip width $(5,5)(w=19.2 \AA)$ in Tables I and II, we found that the two results are basically the same,

TABLE II. Optical transition energies $\Delta E_{n n^{\prime}}$ (in units of eV) and transition matrix elements $Q_{n n^{\prime}}$ (in units of eV) for Si quantum tips with different widths $(m, n)$.

\begin{tabular}{ccllllll}
\hline \hline$(6,6)$ & $\Delta E_{n n^{\prime}}$ & & 3.18 & & 3.28 & & 3.40 \\
& $Q_{n n^{\prime}}$ & & 0.505 & & 0.701 & & 0.617 \\
$(6,5)$ & $\Delta E_{n n^{\prime}}$ & 2.99 & 3.20 & 3.25 & 3.29 & & 3.39 \\
& $Q_{n n^{\prime}}$ & 0.180 & 0.227 & 0.205 & 0.219 & & 0.747 \\
$(5,5)$ & $\Delta E_{n n^{\prime}}$ & 3.05 & 3.17 & 3.25 & 3.26 & 3.31 & 3.40 \\
& $Q_{n n^{\prime}}$ & 0.139 & 0.437 & 0.392 & 0.331 & 1.731 & 0.648 \\
$(6,4)$ & $\Delta E_{n n^{\prime}}$ & 3.01 & 3.20 & 3.26 & 3.28 & 3.30 & 3.35 \\
& $Q_{n n^{\prime}}$ & 0.190 & 0.434 & 0.207 & 0.218 & 0.536 & 0.369 \\
$(5,4)$ & $\Delta E_{n n^{\prime}}$ & 3.00 & 3.18 & 3.25 & & 3.31 & 3.42 \\
& $Q_{n n^{\prime}}$ & 0.255 & 0.341 & 0.110 & & 0.410 & 0.203 \\
$(4,4)$ & $\Delta E_{n n^{\prime}}$ & 3.06 & & & & & 3.42 \\
& $Q_{n n^{\prime}}$ & 0.314 & & & & & 1.207 \\
\hline \hline
\end{tabular}

only the transition elements are slightly different. That means the transitions come mainly from states of $k_{z}=0$.

The remaining problem is how the excited electron relaxes. For the quantum tip the energy bands of bulk semiconductors become one-dimensional subbands, whose density of states is divergent at the band bottom. This energy band structure will prevent the carrier from relaxing to low energy states through emitting phonons because of energy and momentum conservation, something like the phonon bottleneck effect in the zero-dimensional case. ${ }^{10}$ For the quantum tip there is a possibility of carrier relaxation between the one-dimensional subbands. This is because in the directions perpendicular to the tip the phonon momentum, $q_{x}, q_{y} \approx \pi / w \ll q$ (where $q$ is the phonon total momentum), ${ }^{10}$ the carriers relax mainly by emitting phonons in the $z$ direction. It can be proved that electrons cannot relax in subbands by emitting either optical or acoustic phonons, since they cannot satisfy both energy and momentum conservation simultaneously. In order to relax between subbands, electrons need to find two phonons, one with positive and the other negative $q_{z}$ to satisfy both energy and momentum conservation. Therefore the scattering rates in the bulk are larger than those in the tip. Sakaki ${ }^{11}$ has discussed this for quantum wire transport. The electron relaxation time will be larger than or near to the lifetimes, resulting in optical transitions from excited states. From the constitution of wave functions of excited states we found that they come mainly from the $\Gamma$ conduction band states in bulk Si.

In summary, the ultraviolet emission of Si quantum tips is observed. It is due to the quantum confinement effect. By comparing our results with the theoretical results of the empirical pseudopotential homojunction model it is concluded that the four narrow luminescence peaks observed are from Si quantum tips about $20 \AA$ in width and are covered by $\mathrm{SiO}_{2}$. This emission is reproducible for different arrays, and their wavelengths are far shorter than visible light, which means potential applications in various fields. The shoulder that was observed at $3.00 \mathrm{eV}$ is also a consequence of the quantum confinement effect. The broad peak that was observed at $2.50 \mathrm{eV}$ and the broad background in the 2.7-3.6 $\mathrm{eV}$ range are characteristic of an emission from a defect state, and it is believed to be due to the surface state.

This project was funded by a research grant from the Hong Kong Research Grant Council.

${ }^{1}$ L. T. Canham, Appl. Phys. Lett. 57, 1046 (1990).

${ }^{2}$ See, for example, Mater. Res. Soc. Symp. Proc. 256 (1992); 283 (1993); 358 (1995); 452 (1997).

${ }^{3}$ K. Kim, Phys. Rev. B 57, 13072 (1998).

${ }^{4}$ M. Rakhshandehroo and S. W. Pang, J. Vac. Sci. Technol. B 14, 3697 (1996).

${ }^{5}$ M. R. Rakhshandehroo and S. W. Pang, J. Vac. Sci. Technol. B 15, 2777 (1997).

${ }^{6}$ J. B. Xia and K. W. Cheah, Phys. Rev. B 55, 15688 (1997).

${ }^{7} \mathrm{P}$. Balk, The $\mathrm{Si}-\mathrm{SiO}_{2}$ System (Elsevier, Amsterdam, 1988).

${ }^{8}$ S. L. Zhang, K. S. Ho, Y. T. Hou, B. D. Qian, P. Diao, and S. M. Cai, Appl. Phys. Lett. 62, 642 (1993).

${ }^{9}$ X. Wang, D. M. Huang, L. Ye, M. Yang, P. H. Hao, H. X. Fu, X. Y. Hou, and X. D. Xie, Phys. Rev. Lett. 71, 1265 (1993).

${ }^{10}$ H. Benisty, C. M. Sotomayor-Torres, and C. Weisbuch, Phys. Rev. B 44, 10945 (1991).

${ }^{11}$ H. Sakaki, Jpn. J. Appl. Phys., Part 2 19, L735 (1980). 